Research Article

\title{
$L$-Fuzzy Semiprime Ideals of a Poset
}

\author{
Berhanu Assaye Alaba ${ }^{1}$ and Derso Abeje Engidaw $\mathbb{D}^{2}$ \\ ${ }^{1}$ Department of Mathematics, Bahir Dar University, Bahir Dar, Ethiopia \\ ${ }^{2}$ Department of Mathematics, University of Gondar, Gondar, Ethiopia \\ Correspondence should be addressed to Derso Abeje Engidaw; deab02@yahoo.com
}

Received 6 January 2020; Revised 13 February 2020; Accepted 14 May 2020; Published 31 May 2020

Academic Editor: Ping-Feng Pai

Copyright (C) 2020 Berhanu Assaye Alaba and Derso Abeje Engidaw. This is an open access article distributed under the Creative Commons Attribution License, which permits unrestricted use, distribution, and reproduction in any medium, provided the original work is properly cited.

In this paper, we introduce the concept of $L$-fuzzy semiprime ideal in a general poset. Characterizations of $L$-fuzzy semiprime ideals in posets as well as characterizations of an $L$-fuzzy semiprime ideal to be $L$-fuzzy prime ideal are obtained. Also, $L$-fuzzy prime ideals in a poset are characterized.

\section{Introduction}

Fuzzy set theory was introduced by Zadeh in 1965 as an extension of the classical notion of set theory [1]. In 1971, Rosenfeld wrote his seminal paper on fuzzy subgroups in [2]. This paper has provided sufficient motivations for researchers to study the fuzzy subalgebras of different algebraic structures, like rings, modules, vector-spaces, lattices, and more recently in MS-algebras, universal algebras, pseudocomplemented semilattice, and so on (see [3-26]).

Zadeh defined a fuzzy subset of a nonempty set $X$ as a function from $X$ to unit interval $[0,1]$ of real numbers. Goguen in [27] generalized the fuzzy subsets of $X$, to L-fuzzy subsets, as a function from $X$ to a lattice $L$. Swamy and Swamy [5] initiated that complete lattices satisfying the infinite meet distributivity are the most appropriate candidates to have the truth values of general fuzzy statements.

In the literature, we have found several types of ideals and filters of a poset which are generalizations of ideals and filters of a lattice (see [28-33]). Halaś and Rachůnek in [34] introduced the notions of prime ideals in a poset, and Khart and Mokbel [35] introduced the concept of a semiprime ideal in general poset.

In $[36,37]$, the authors of this paper introduced several types of $L$-fuzzy ideals and filters of a partially ordered set whose truth values are in a complete lattice satisfying the infinite meet distributive law. In addition, in [38], we have introduced and presented certain comprehensive results on the notion of $L$-fuzzy prime ideals and maximal $L$-fuzzy ideals of a poset by applying the general theory of algebraic fuzzy systems introduced in $[39,40]$.

Initiated by the above ideas and concepts, in this paper, we introduce and develop the concepts of $L$-fuzzy semiprime ideal in a general partially ordered set. Characterizations of $L$-fuzzy semiprime ideals in posets as well as necessary and sufficient conditions of an $L$-fuzzy semiprime ideal to be $L$-fuzzy prime ideal are observed. Also, by introducing the concept of a $\mu$-atom element in a poset, we obtain characterizations of $L$-fuzzy semiprime ideals and $L$-fuzzy prime ideals in a poset satisfying the descending chain condition (DCC).

\section{Preliminaries}

For the necessary concepts, terminologies, and notations, we refer to $[41,42]$.

A pair $(Q, \leq)$ is called a partially ordered set or simply a poset if $Q$ is a nonempty set and " $\leq$ " is a partial order on $Q$. An element $x \in Q$ is called a lower bound of $S$ if $x \leq s$ for all $s \in S$. An upper bound is defined dually. The set of all lower bounds of $S$ is denoted by $S^{l}$ and the set of all upper bounds of $S$, by $S^{u}$.

By the sets $S^{u l}$ and $S^{l u}$, we mean $\left\{S^{u}\right\}^{l}$ and $\left\{S^{l}\right\}^{u}$, respectively. For any $a, b \in Q$, the sets $\{a\}^{l}$ and $\{a, b\}^{l}$ are 
denoted by $a^{l}$ and $(a, b)^{l}$, respectively. Furthermore, for subsets $S, T$ of $Q,\{S \cup T\}^{l}$ is denoted by $\{S, T\}^{l}$ and the set $\{S \cup\{a\}\}^{l}$ is denoted by $\{S, a\}^{l}$. Similar notations are used for the set of all upper bounds of subsets of a poset $Q$.

For any subsets $S, T$ of a poset $Q$, we note that $S \subseteq S^{u l}$ and $S \subseteq S^{l u}$, and if $S \subseteq T$ in $Q$, then $S^{u} \supseteq T^{u}$ and $S^{l} \supseteq T^{l}$. In addition, $\left\{a^{u}\right\}^{l}=a^{l}$ and $\left\{a^{l}\right\}^{u}=a^{u}$. An element $x_{0}$ in $Q$ is called the greatest lower bound of $S$ or infimum of $S$, denoted by infS, if $x_{0} \in S^{l}$ and $x \leq x_{0} \forall x \in S^{l}$. Dually, we have the concept of the least upper bound of $S$ or supremum of $S$ which is denoted by supS.

For $x, y \in Q$, we write $x \wedge y$ (read as " $x$ meet $y$ ") in place of $\inf \{x, y\}$ if it exists and $x \vee y$ (read as " $x$ join $y$ ") in place of $\sup \{x, y\}$ if it exists. An element $q_{0}$ in $Q$ is called the smallest (respectively, the largest) element of a poset $Q$ if $q_{0} \leq x$ (respectively, $x \leq q_{0}$ ) for all $x \in Q$. The smallest (respectively, the largest) element if it exists in $Q$ is denoted by 0 (respectively, by 1$)$. A poset $(Q \leq)$ is called bounded if it has 0 and 1.

A poset $Q$ is is said to satisfy the ascending chain condition (ACC), if every nonempty subset of $Q$ has a maximal element. Dually, we have the concept of descending chain condition (DCC) [35].

Definition 1 (see [43]). A poset $Q$ is called distributive if for all $a, b, c \in Q$,

$$
\left\{(a, b)^{u}, c\right\}^{l}=\left\{(a, c)^{l},(b, c)^{l}\right\}^{u l} .
$$

Definition 2 (see [24]). A subset $I$ of a poset $(Q, \leq)$ is called an ideal in $Q$ if $(a, b)^{u l} \subseteq I$ whenever $a, b \in I$.

Now, we consider the concept of a semiprime ideal introduced by Khart and Mokbel in a poset and by Rav in a lattice, as given in the following.

Definition 3 (see [28]). A proper ideal $I$ of a poset $Q$ is called a semiprime ideal of $Q$ if for all $x, y, z \in Q,(x, y)^{l} \subseteq I$ and $(x, z)^{l} \subseteq I$ imply $\left\{x,(y, z)^{u}\right\}^{l} \subseteq I$. Q.

Dually, we have the concept semiprime filter of a poset

Definition 4 (see [44]). A proper ideal $I$ of a lattice $X$ is called a semiprime ideal of $X$ if for all $x, y, z \in X, x \wedge y \in I$ and $x \wedge z \in I$ together imply $x \wedge(y \vee z) \in I$. $X$.

Dually, we have the concept semiprime filter of a lattice

For an ideal $I$ and an element $a$ in a poset $Q$, define a set $I: a$ by

$$
I: a=\left\{x \in Q:(a, x)^{l} \subseteq I\right\}
$$

Definition 5 (see [28]). An element $i$ in a poset $Q$ is called an $I$-atom with respect to an ideal $I$ of $Q$ if $i \notin I$ and for any $x \in Q$ with $x<i$ implies $x \in I$.

Throughout this paper, $L$ stands for a complete lattice satisfying the infinite meet distributive law and $Q$ stands for a poset with 0 .
By an $L$-fuzzy subset $\mu$ of a poset $Q$, we mean a mapping from $Q$ into $L$. We denote the set of $L$ - fuzzy subsets of $Q$ by $L^{Q}$. For each $\alpha \in L$ and $\mu \in L^{Q}$, the $\alpha$-level subset of $\mu$, which is denoted by $\mu_{\alpha}$, is a subset of $Q$ given by $\mu_{\alpha}=\{x: \mu(x) \geq \alpha\}$. For fuzzy subsets $\mu$ and $\sigma$ of $Q$, we write $\mu \subseteq \sigma$ to mean $\mu(x) \leq \sigma(x)$ for all $x \in Q$ in the ordering of $L$. It can be easily verified that " $\subseteq$ " is a partial order on the set $L^{Q}$ and is called the point-wise ordering. We write $\mu \subset \sigma$ if $\mu \subseteq \sigma$ and $\mu \neq \sigma$.

The following notions and results in this section are from the authors' work in $[29,31]$.

Definition 6. $\mu \in L^{Q}$ is said to be an $L$ - fuzzy semi-ideal of $Q$ if $\mu(0)=1$ and for any $a \in Q, \mu(x) \geq \mu(a)$, for all $x \in a^{l}$.

Definition 7. $\mu \in L^{Q}$ is said to be an $L$ - fuzzy ideal of $Q$ if $\mu(0)=1$ and, for any, $a, b \in Q$,

$$
\mu(x) \geq \mu(a) \wedge \mu(b), \quad \text { for all } x \in(a, b)^{u l} .
$$

An $L$-fuzzy ideal $\mu$ of $Q$ is called a $u$ - $L$-fuzzy ideal if, for any $a, b \in Q$, there exists $x \in(a, b)^{u}$ such that $\mu(x)=$ $\mu(a) \wedge \mu(b)$.

Lemma 1. $\mu \in L^{Q}$ is an L-fuzzy ideal of $Q$ if and only if $\mu_{\alpha}$ is an ideal of $Q$, for all $\alpha \in L$.

Lemma 2. If $\mu$ is an $L$-fuzzy ideal of $Q$, then $\mu$ is anti-tone. Note that, for any $\beta$ in $L$ the constant $L$-fuzzy subset of $Q$ which maps all elements of $Q$ onto $\beta$, is denoted by $\bar{\beta}$.

Definition 8. An $L$-fuzzy ideal $\mu$ of a poset $Q$ is called proper, if $\mu \neq \overline{1}$, where 1 is the largest element in $L$.

Definition 9. A proper $L$-fuzzy ideal $\mu$ of a poset $Q$ is called an $L$-fuzzy prime, if, for any $a, b \in Q$,

$$
\inf \left\{\mu(x): x \in(a, b)^{l}\right\}=\mu(a) \text {, or } \mu(b) .
$$

Definition 10. A proper $L$-fuzzy ideal $\mu$ of a poset $Q$ is said to be maximal if $\mu$ is a maximal element in the set of all proper $L$-fuzzy ideals of $Q$.

\section{L-Fuzzy Semiprime Ideals of a Poset}

In this section, we introduce and develop the notions of L-fuzzy semiprime ideal of a poset and give several characterizations of it. We shall begin with its definition.

Definition 11. An $L$-fuzzy ideal $\mu$ of a poset $Q$ is called an $L$-fuzzy semiprime ideal if for all $a, b, c \in Q$,

$$
\mu(z) \geq \inf \left\{\mu(x) \wedge \mu(y): x \in(a, b)^{l}, y \in(a, c)^{l}\right\}, \quad \forall z \in\left\{a,(b, c)^{u}\right\}^{l} .
$$

The following result characterizes any $L$-fuzzy semiprime ideal of $Q$ in terms of its level subsets. 
Lemma 3. An L-fuzzy ideal $\mu$ of $Q$ is an L-fuzzy semiprime ideal of $Q$ if and only if $\mu_{\alpha}$ is a semiprime ideal of $Q$ for all $\alpha \in L$.

Proof. Suppose that $\mu$ is an $L$-fuzzy semiprime ideal and $\alpha \in L$. Then, clearly, $\mu_{\alpha}$ is an ideal of $Q$. Let $a, b, c \in Q$ such that $(a, b)^{l} \subseteq \mu_{\alpha}$ and $(a, c)^{l} \subseteq \mu_{\alpha}$ and $z \in\left\{a,(b, c)^{u}\right\}^{l}$. Then, $\mu(x) \geq \alpha \forall x \in(a, b)^{l}$ and $\mu(y) \geq \alpha \forall y \in(a, b)^{l}$. This implies that

$$
\begin{aligned}
& \inf \left\{\mu(x): x \in(a, b)^{l}\right\} \geq \alpha, \\
& \inf \left\{\mu(y): y \in(a, c)^{l}\right\} \geq \alpha .
\end{aligned}
$$

Therefore, $\inf \left\{\mu(x) \wedge \mu(y): x \in(a, b)^{l}, y \in(a, c)^{l}\right\} \geq \alpha$. Since $\mu$ is an $L$-fuzzy semiprime ideal and $z \in\left\{a,(b, c)^{u}\right\}^{l}$, we have

$$
\mu(z) \geq \inf \left\{\mu(x) \wedge \mu(y): x \in(a, b)^{l}, y \in(a, c)^{l}\right\} \geq \alpha .
$$

This implies that $z \in \mu_{\alpha}$ for all $z \in\left\{a,(b, c)^{u}\right\}^{l}$ and hence, $\left\{a,(b, c)^{u}\right\}^{l} \subseteq \mu_{\alpha}$. Therefore, $\mu_{\alpha}$ is a semiprime ideal of a poset $Q$.

Conversely, suppose that $\mu_{\alpha}$ is a semiprime ideal of $Q$ for all $\alpha \in L$. Then, clearly, $\mu$ is an $L$ - fuzzy ideal of $Q$. Let $a, b, c \in Q$ and put

$$
\alpha=\inf \left\{\mu(x) \wedge \mu(y): x \in(a, b)^{l}, y \in(a, c)^{l}\right\} .
$$

Then,

$$
\begin{aligned}
& \inf \left\{\mu(x): x \in(a, b)^{l}\right\} \geq \alpha, \\
& \inf \left\{\mu(y): y \in(a, c)^{l}\right\} \geq \alpha .
\end{aligned}
$$

That is, $\mu(x) \geq \alpha \forall x \in(a, b)^{l}$ and $\mu(y) \geq \alpha \forall y \in(a, c)^{l}$. This implies that $(a, b)^{l} \subseteq \mu_{\alpha}$ and $(a, c)^{l} \subseteq \mu_{\alpha}$. Thus, since $\mu_{\alpha}$ is a semiprime ideal of $Q$, we have $\left\{a,(b, c)^{u}\right\} \subseteq \mu_{\alpha}$. Therefore, $\mu(z) \geq \alpha=\inf \left\{\mu(x) \wedge \mu(y): x \in(a, b)^{l} x \in(a, c)^{l}\right\}$ for all $z \in\left\{a,(b, c)^{u}\right\}^{l}$, and hence $\mu$ is an $L$-fuzzy semiprime ideal of Q.

Corollary 1. A subset I of a poset $Q$ is a semiprime ideal of $Q$ if and only if its characteristic map $\chi_{I}$ is an L-fuzzy semiprime ideal of $Q$.

Definition 12. An $L$-fuzzy ideal $\mu$ of a lattice $Q$ is called an $L$-fuzzy semiprime ideal, if for all $a, b, c \in Q$,

$$
\mu(a \wedge(b \vee c))=\mu(a \wedge b) \wedge \mu(a \wedge c) .
$$

Dually, we have the concept of $L$-fuzzy semiprime filter of a lattice $Q$.

Lemma 4. Let $\mu$ be an L-fuzzy ideal of $Q$. Then, for any $a, b \in Q$,

$$
\inf \left\{\mu(x): x \in(a, b)^{l}\right\}=\mu(a \wedge b),
$$

whenever $a \wedge b$ exists in $Q$.

The following theorem shows that an L-fuzzy semiprime ideal of a poset is a natural generalization of an L-fuzzy semiprime ideal of a lattice.
Theorem 1. Let $(Q, \leq)$ be a lattice. Then, an L-fuzzy ideal of $Q$ is an $L$-fuzzy semiprime ideal in the poset $Q$ if and only if it is an L-fuzzy semiprime ideal in the lattice $Q$.

Proof. Let $\mu$ be an $L$-fuzzy semiprime ideal in the poset $Q$ and $a, b, c \in Q$. Then, since $a \wedge(b \vee c) \in\left\{a,(b, c)^{u}\right\}^{l}$, we have

$$
\begin{aligned}
\mu(a \wedge(b \vee c)) & \geq \inf \left\{\mu(x) \wedge \mu(y): x \in(a, b)^{l}, y \in(a, c)^{l}\right\} \\
& =\inf \left\{\mu(x): x \in(a, b)^{l}\right\} \wedge \inf \left\{\mu(y): y \in(a, c)^{l}\right\} \\
& =\mu(a \wedge b) \wedge \mu(a \wedge c) .
\end{aligned}
$$

Again, since $a \wedge b \leq a \wedge(b \vee c), a \wedge c \leq a \wedge(b \vee c)$ and $\mu$ is antitone, we clearly have

$$
\mu(a \wedge(b \vee c)) \leq \mu(a \wedge b) \wedge \mu(a \wedge c) .
$$
Q.

Therefore, $\mu$ is an $L$-fuzzy semiprime ideal in the lattice

Conversely, suppose that $\mu$ is an $L$-fuzzy semiprime ideal in the lattice $Q$. Let $a, b, c \in Q$ and $z \in\left\{a,(b, c)^{u}\right\}^{l}$. Then, $z \leq a$ and $z \leq t$, for all $t \in(a, b)^{u}$. Since $a \vee b \in(a, b)^{u}$, we have $z \leq a \vee b$. This implies that $z \leq a \wedge(b \vee c)$ and hence

$$
\begin{aligned}
\mu(z) & \geq \mu(a \wedge(b \vee c)) \\
& \geq \mu(a \wedge b) \wedge \mu(a \wedge c) \\
& =\inf \left\{\mu(x): x \in(a, b)^{l}\right\} \wedge \inf \left\{\mu(y): y \in(a, c)^{l}\right\} \\
& =\inf \left\{\mu(x) \wedge \mu(y): x \in(a, b)^{l}, y \in(a, c)^{l}\right\} .
\end{aligned}
$$

So, $\mu$ is an $L$-fuzzy semiprime ideal in the poset $Q$.

The following result establishes a connection between $L$-fuzzy prime ideals and $L$-fuzzy semiprime ideals of a poset $Q$.

Lemma 5. Every L-fuzzy prime ideal of a poset $Q$ is an L-fuzzy semiprime ideal.

Proof. Let $\mu$ be an $L$-fuzzy prime ideal of $Q$. Let $a, b, c \in Q$. Then since $\mu$ is an $L$-fuzzy prime ideal of $Q$, we clearly have

$$
\begin{array}{r}
\inf \left\{\mu(x): x \in(a, b)^{l}\right\}=\mu(a), \\
\text { or } \mu(b), \\
\inf \left\{\mu(y): y \in(a, c)^{l}\right\}=\mu(a), \\
\text { or } \mu(c) .
\end{array}
$$

Let $z \in\left\{a,(b, c)^{u}\right\}^{l}=a^{l} \cap(b, c)^{u l}$. Then, $z \leq a$ and $z \in(b, c)^{u l}$. Now if $\inf \left\{\mu(x): x \in(a, b)^{l}\right\}=\mu(a)$ or $\inf \left\{\mu(y): y \in(a, c)^{l}\right\}=\mu(a)$, then we have

$$
\begin{aligned}
\mu(z) & \geq \mu(a) \geq \inf \left\{\mu(x): x \in(a, b)^{l}\right\} \wedge \inf \left\{\mu(y): y \in(a, c)^{l}\right\} \\
& =\inf \left\{\mu(x) \wedge \mu(y): x \in(a, b)^{l}, y \in(a, c)^{l}\right\} .
\end{aligned}
$$

Again if $\inf \left\{\mu(x): x \in(a, b)^{l}\right\} \neq \mu(a)$ and $\inf \{\mu(y):$ $\left.y \in(a, c)^{l}\right\} \neq \mu(a)$, then we have 


$$
\begin{aligned}
& \inf \left\{\mu(x): x \in(a, b)^{l}\right\}=\mu(b), \\
& \inf \left\{\mu(y): y \in(a, c)^{l}\right\}=\mu(c) .
\end{aligned}
$$

Now since $z \in(b, c)^{u l}$ and $\mu$ is an $L$-fuzzy ideal, we have

$$
\begin{aligned}
\mu(z) & \geq \mu(b) \wedge \mu(c)=\inf \left\{\mu(x): x \in(a, b)^{l}\right\} \wedge\left\{\mu(y): y \in(a, c)^{l}\right\} \\
& =\inf \left\{\mu(x) \wedge \mu(y): x \in(a, b)^{l}, y \in(a, c)^{l}\right\} .
\end{aligned}
$$

Hence, in either cases, we have

$$
\mu(z) \geq \inf \left\{\mu(x) \wedge \mu(y): x \in(a, b)^{l}, y \in(a, c)^{l}\right\}, \quad \text { for all } z \in\left\{a,(b, c)^{u}\right\}^{l}
$$

Hence, $\mu$ is an $L$-fuzzy semiprime ideal of $Q$.

Remark 1. The converse of the above lemma is not true. For example, consider the poset $(Q \leq)$ depicted in Figure 1. Define a fuzzy subset $\mu: Q \longrightarrow[0,1]$ by

$$
\begin{aligned}
& \mu(0)=1, \\
& \mu(a)=\mu(b)=\mu(1)=0.5 .
\end{aligned}
$$

Then, $\mu$ is an $L$-fuzzy semiprime ideal but not an $L$-fuzzy prime ideal of $Q$. This is because $a, b \in Q$ and $\inf \left\{\mu(x): x \in(a, b)^{l}\right\}=\mu(0)=1 \neq 0.5=\mu(a)=\mu(b)$.

Now, given an $L$-fuzzy ideal of a poset $Q$ and any element in $Q$, we define the following $L$-fuzzy subset of $Q$.

Definition 13. Let $\mu$ be an $L$-fuzzy ideal of $Q$ and $x \in Q$. Define an $L$-fuzzy subset $\mu: x$ of $Q$ by

$$
(\mu: x)(y)=\inf \left\{\mu(z): z \in(x, y)^{l}\right\}, \quad \text { for all } z \in Q .
$$

From Definition 9, observe that an $L$-fuzzy ideal $\mu$ of $Q$ is an $L$-fuzzy prime ideal if, for any $a, b \in Q$,

$$
(\mu: a)(b)=\mu(a), \text { or } \mu(b) .
$$

Now, we have the following lemmas.

Lemma 6. Let $\mu$ be an L-fuzzy ideal of $Q$ and $x \in Q$. Then, $\mu: x$ is an L-fuzzy semi-ideal containing $\mu$.

Proof. Now

$$
\begin{aligned}
(\mu: x)(0) & =\inf \left\{\mu(z): z \in(x, 0)^{l}\right\} \\
& =\inf \{\mu(z): z=0\} \\
& =\mu(0)=1 .
\end{aligned}
$$

Therefore, $(\mu: x)(0)=1$. Again, let $a \in Q$ and $y \in a^{l}$. Now

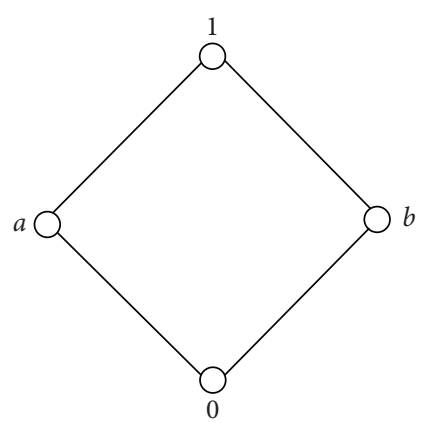

Figure 1: An $L$-fuzzy semi-prime ideal which is not an $L$-fuzzy prime ideal of $Q$.

$$
\begin{aligned}
(\mu: x)(y) & =\inf \left\{\mu(w): w \in(x, y)^{l}\right\} \\
& \geq \inf \left\{\mu(w): w \in(x, a)^{l}\right\}\left(\operatorname{since}(x, y)^{l} \subseteq(x, a)^{l}\right) \\
& =(\mu: x)(a) .
\end{aligned}
$$

Therefore, $\mu: x$ is an $L$-fuzzy semi-ideal. Again, for all $y \in Q$, we have

$$
(\mu: x)(y)=\inf \left\{\mu(z): z \in(x, y)^{l}\right\} \geq \mu(y) .
$$

Hence, $\mu \subseteq \mu: x$.

Note that, for any $x, y \in Q$, observe that $(\mu: x)(y)=(\mu: y)(x)$.

Remark 2. For an $L$-fuzzy ideal $\mu$ of a poset $Q \mu: x$ need not be an $L$-fuzzy ideal of $Q$ for all $x \in Q$. For example, consider the poset $(Q, \leq)$ depicted in Figure 2 .

Define a fuzzy subset $\mu: Q \longrightarrow[0.1]$ by

$$
\begin{aligned}
& \mu(0)=1, \\
& \mu(a)=0.8, \\
& \mu(b)=\mu(c)=\mu(d)=\mu(e)=0.2 .
\end{aligned}
$$

Then, $\mu$ is an $L$-fuzzy ideal of $Q$, and $\mu: d$ is a fuzzy subset of $Q$ given by

$$
\begin{aligned}
& (\mu: d)(0)=(\mu: d)(b)=(\mu: d)(c)=1, \\
& (\mu: d)(a)=0.8 \\
& (\mu: d)(d)=(\mu: d)(e)=0.2 .
\end{aligned}
$$

Observe that $e \in(a, b)^{u l}$ but $(\mu: d)(e)=$ $0.2 \ngtr 0.8=(\mu: d)(a) \wedge(\mu: d)(b)$. This implies that $\mu: d$ is not an $L$-fuzzy ideal of $Q$.

Lemma 7. Let $\mu$ be an L-fuzzy ideal of a poset $Q$ and $x \in Q$. Then,

$$
(\mu: x)_{\alpha}=\mu_{\alpha}: x, \quad \text { for any } \alpha \in L
$$




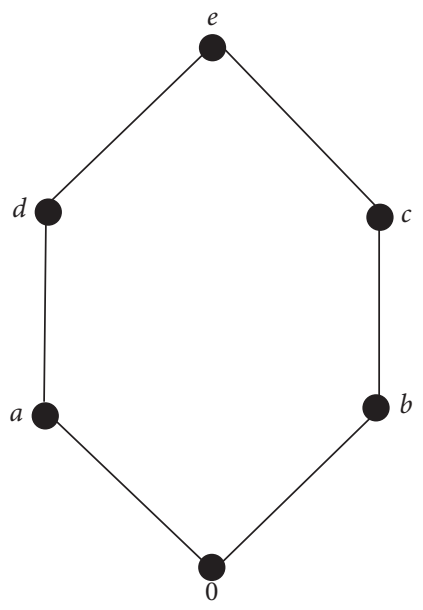

Figure 2: An L -fuzzy ideal $\mu$ of a poset $Q$ for which $\mu$ : $x$ need not to be L -fuzzy ideal for $x \in Q$.

Proof. Now, we have

$$
\begin{aligned}
y \in(\mu: x)_{\alpha} & \Leftrightarrow(\mu: x)(y) \geq \alpha \\
& \Leftrightarrow \inf \left\{\mu(w): w \in(x, y)^{l}\right\} \geq \alpha \\
& \Leftrightarrow \mu(w) \geq \alpha \forall w \in(x, y)^{l} \\
& \Leftrightarrow w \in \mu_{\alpha} \forall w \in(x, y)^{l} \\
& \Leftrightarrow(x, y)^{l} \subseteq \mu_{\alpha} \\
& \Leftrightarrow y \in \mu_{\alpha}: x .
\end{aligned}
$$

Therefore $(\mu: x)_{\alpha}=\mu_{\alpha}: x$.

Lemma 8. Let $\mu$ be an L-fuzzy ideal of a poset $Q$ and $x \in Q$. Then, the following hold: (1) $\inf _{\left.b)^{l}\right\}}\left\{(\mu: x)(y): y \in(a, b)^{l}\right\}=\inf \{\mu(y): y \in(x, a$,

(2) $\inf _{\left.\left.b)^{u}\right\}^{l}\right\}}\left\{(\mu: x)(y): y \in(a, b)^{u l}\right\}=\inf \{\mu(y): y \in\{x,(a$,

(3) $\mu: x=\overline{1}$ if and only if $\mu(x)=1$

\section{Proof}

(1) Put $A=\left\{\mu(y): y \in(x, a, b)^{l}\right\}$ and $B=\{(\mu: x)(y): y$ $\left.\in(a, b)^{l}\right\}$. Now we claim that $\inf A=\inf B$. Let $\alpha=\inf A$. Then,

$$
\begin{aligned}
\alpha \leq \mu(y), \quad \forall y \in(x, a, b)^{l} & \Longrightarrow(x, a, b)^{l} \subseteq \mu_{\alpha} \\
& \Longrightarrow(a, b)^{l} \subseteq \mu_{\alpha}: x=(\mu: x)_{\alpha} \\
& \Longrightarrow(\mu: x)(y) \geq \alpha, \quad \forall y \in(a, b)^{l} \\
& \Longrightarrow \inf \left\{(\mu: x)(y): y \in(a, b)^{l}\right\} \geq \alpha . \\
& \Longrightarrow \inf B \geq \inf A .
\end{aligned}
$$

To prove the other side of the inequality, let $\beta=\inf B$. Then,

$$
\begin{aligned}
\beta \leq(\mu: x)(y), \quad \forall y \in(a, b)^{l} & \Longrightarrow(a, b)^{l} \subseteq(\mu: x)_{\beta}=\mu_{\beta}: x \\
& \Longrightarrow(x, a, b)^{l} \subseteq \mu_{\beta} \\
& \Longrightarrow \mu(y) \geq \beta, \quad \forall y \in(x, a, b)^{l} \\
& \Longrightarrow \inf \left\{\mu(y): y \in(x, a, b)^{l}\right\} \geq \beta \\
& \Longrightarrow \inf A \geq \inf B .
\end{aligned}
$$

Hence, the claim is true.

(2) The proof is similar to 1.

(3) Suppose $\mu: x=\overline{1}$. Then, $(\mu: x)(y)=1$, for all $y \in Q$.

Thus, in particular, $(\mu: x)(x)=1$ :

$$
\begin{aligned}
\operatorname{now}(\mu: x)(x)=1 & \Rightarrow \inf \left\{\mu(y): y \in(x, x)^{l}\right\}=1 \\
& \Rightarrow \mu(y)=1, \quad \forall y \in(x, x)^{l} \\
& \Rightarrow \mu(x)=1 \ldots\left(\text { since } x \in(x, x)^{l}\right) .
\end{aligned}
$$

Conversely suppose that $\mu(x)=1$. Now since, for any $y \in Q, \quad(\mu: x)(y)=\inf \left\{\mu(z): z \in(x, y)^{l}\right\} \geq \mu(x)=1$, we have $(\mu: x)(y)=1$ for all $y \in Q$. Therefore, $\mu: x=\overline{1}$.

Now, we present a characterization of an $L$-fuzzy semiprime ideal of a poset $Q$ in terms of $\mu: x$ where $\mu$ is an $L$-fuzzy ideal of $Q$ and $x \in Q$.

Theorem 2. An L-fuzzy ideal $\mu$ of a poset $Q$ is an L-fuzzy semiprime ideal if and only if $\mu: x$ is an L-fuzzy ideal for all $x \in Q$, in fact, an $L$-fuzzy semiprime ideal for all $x \in Q$.

Proof. Let $\mu$ be an $L$-fuzzy semiprime ideal of $Q$ and $x \in Q$. First, let us show that $\mu: x$ is an $L$-fuzzy ideal of $Q$. Since $(\mu: x)(0)=\inf \left\{\mu(y): y \in(x, 0)^{l}\right\}=\mu(0)=1$, we have $(\mu: x)(0)=1$. Again, let $a, b \in Q$ and $z \in(a, b)^{u l}$. Then, 


$$
\begin{aligned}
(\mu: x)(a) \wedge(\mu: x)(b) & =\inf \left\{\mu(w): w \in(x, a)^{l}\right\} \wedge \inf \left\{\mu(u): u \in(x, b)^{l}\right\} \\
& =\inf \left\{\mu(w) \wedge \mu(u): w \in(x, a)^{l}, u \in(x, b)^{l}\right\} \\
& \leq \mu(v) \text { for all } v \in\left\{x,(a, b)^{u}\right\}^{l}
\end{aligned}
$$

Again, since $z \in(a, b)^{u l}$ implies $(x, z)^{l} \subseteq\left\{x,(a, b)^{u}\right\}^{l}$, we have $(\mu: x)(a) \wedge(\mu: x)(b) \leq \mu(t)$ for all $t \in(x, z)^{l}$. This implies that

$$
(\mu: x)(a) \wedge(\mu: x)(b) \leq \inf \left\{\mu(t): t \in(x, z)^{l}\right\}=(\mu: x)(z) .
$$

Therefore, $\mu: x$ is an $L$-fuzzy ideal of $Q$ for all $x \in Q$. Now, we show that $\mu: x$ is an $L$-fuzzy semiprime ideal of $Q$. Let $a, b, c \in Q$ and $z \in\left\{a,(b, c)^{u}\right\}^{l}$. Now,

$$
\begin{aligned}
\inf & \left\{(\mu: x)(u) \wedge(\mu: x)(w): u \in(a, b)^{l}, w \in(a, c)^{l}\right\} \\
& =\inf \left\{(\mu: x)(u): u \in(a, b)^{l}\right\} \wedge \inf \left\{(\mu: x)(w): w \in(a, c)^{l}\right\} \\
& =\inf \left\{\mu(u): u \in(x, a, b)^{l}\right\} \wedge \inf \left\{\mu(w): w \in(x, a, c)^{l}\right\} \\
& =\inf \left\{(\mu: b)(u): u \in(x, a)^{l}\right\} \wedge \inf \left\{(\mu: c)(w): w \in(x, a)^{l}\right\} \\
& =\inf \left\{(\mu: b)(s) \wedge(\mu: c)(s): s \in(x, a)^{l}\right\} \\
& \leq \inf \left\{(\mu: b)(s) \wedge(\mu: c)(s): s \in\left\{x, a,(b, c)^{u}\right\}^{l}\right\} \\
& \leq(\mu: b)(s) \wedge(\mu: c)(s) \forall s \in\left\{x, a,(b, c)^{u}\right\}^{l}=(x, a)^{l} \cap(b, c)^{u l} \\
& =(\mu: s)(b) \wedge(\mu: s)(c) \forall s \in\left\{x, a,(b, c)^{u}\right\}^{l} \\
& =(\mu: s)(s) \forall s \in\left\{x, a,(b, c)^{u}\right\}^{l} \\
& =\mu(s) \forall s \in\left\{x, a,(b, c)^{u}\right\}^{l} .
\end{aligned}
$$

Now, $z \in\left\{a,(b, c)^{u}\right\}^{l}$ implies that $(x, z)^{l} \subseteq\left\{x, a,(b, c)^{u}\right\}^{l}$ for all $x \in Q$. Thus, we have

$$
\begin{aligned}
& \inf \left\{(\mu: x)(u) \wedge(\mu: x)(w): u \in(a, b)^{l}, w \in(a, c)^{l}\right\} \\
& \quad \leq \mu(s), \quad \text { for all } s \in(x, z)^{l} .
\end{aligned}
$$

Thus, $\quad \inf \left\{(\mu: x)(u) \wedge(\mu: x) \quad(w): u \in(a, b)^{l}\right.$, $\left.w \in(a, c)^{l}\right\} \leq \inf \left\{\mu(s): s \in(x, z)^{l}\right\}=(\mu: x)(z)$. Therefore, $\mu: x$ is an $L$-fuzzy semiprime ideal of $Q$.

Conversely, suppose that $\mu: x$ is an $L$-fuzzy ideal of $Q$ for all $x \in Q$. Now we show that $\mu$ is an $L$-fuzzy semiprime ideal of $Q$. Let $a, b, c \in Q$ and $z \in\left\{a,(b, c)^{u}\right\}^{l}$. Then,

$$
\begin{aligned}
\inf & \left\{\mu(x) \wedge \mu(y): x \in(a, b)^{l}, y \in(a, c)^{l}\right\} \\
& =\inf \left\{\mu(x): x \in(a, b)^{l}\right\} \wedge \inf \left\{\mu(y): y \in(a, c)^{l}\right\} \\
& =(\mu: a)(b) \wedge(\mu: a)(c) \\
& \leq(\mu: a)(z), \quad \text { for all } z \in(b, c)^{u l} .
\end{aligned}
$$

This implies that

$$
\begin{aligned}
\inf \left\{\mu(x) \wedge \mu(y): x \in(a, b)^{l}, y \in(a, c)^{l}\right\} & \leq \inf \left\{(\mu: a)(z): z \in(a, b)^{u l}\right\} \\
& =\inf \left\{\mu(z): z \in\left\{a,(b, c)^{u}\right\}^{l}\right\} .
\end{aligned}
$$

Thus,

$$
\inf \left\{\mu(x) \wedge \mu(y): x \in(a, b)^{l}, y \in(a, c)^{l}\right\} \leq \mu(z), \quad \text { for all } z \in\left\{a,(b, c)^{u}\right\}^{l}
$$

$$
(\mu: a)(b)=\mu(a), \text { or } \mu(b) .
$$

Therefore, $\mu$ is an $L$-fuzzy semiprime ideal of $Q$.

The next result is a characterization of an $L$-fuzzy ideal to be an $L$-fuzzy prime ideal in a poset $Q$.

Theorem 3. Let $\mu$ be a proper L-fuzzy ideal of a poset $Q$. Then, $\mu$ is an L-fuzzy prime ideal of $Q$ if and only if $\mu: a=\mu$ for all $a \in Q$ such that $\mu(a) \neq 1$.

Proof. Suppose that $\mu$ is an $L$-fuzzy prime ideal of $Q$ and let $a \in Q$ such that $\mu(a) \neq 1$. Then, by Lemma 5 and Theorem 2 , it is clear that $\mu: a$ is an $L$-fuzzy ideal of $Q$. Now we claim that $\mu: a=\mu$. Now, for any $x \in Q$, we have $(\mu: a)(x)=$ $\mu(x)$ or $\mu(a)$. However, as $\mu: a$ is an $L$-fuzzy ideal of $Q,(\mu$ : a) $(x) \neq \mu(a)$. Thus, $(\mu: a)(x)=\mu(x)$ for all $x \in Q$ and hence $\mu: a=\mu$.

Conversely, suppose that the given condition holds. Let $a, b \in Q$. Now, we claim that
Suppose that $(\mu: a)(b) \neq \mu(a)$. Then, $\inf \{\mu(x)$ : $\left.x \in(a, b)^{l}\right\} \not \mu(a)$. This implies that $\mu(a) \neq 1$. Thus, by hypothesis, we have $\mu: a=\mu$ and hence $(\mu: a)(b)=\mu(b)$.

Therefore, $\mu$ is an $L$-fuzzy prime ideal of $Q$.

Now before we prove some other characterizations of $L$-fuzzy primeness and $L$-fuzzy semiprimeness in the case of a poset satisfying DCC, we introduce the concept of a $\mu$-atom of an $L$-fuzzy ideal $\mu$ of a poset.

Definition 14. Let $\mu$ be an $L$-fuzzy ideal of a poset $Q$ and $\alpha \in L$. An element $i$ in $Q$ is called a $\mu$-atom with respect to $\alpha$, if it satisfies the following conditions:

(1) $\alpha \not \mu(i)$

(2) $\alpha \leq \mu(x)$ whenever $x<i$ 
Example 1. Consider the poset depicted in Figure 3. Define a fuzzy subset $\mu: Q \longrightarrow[0,1]$ by

$$
\begin{aligned}
\mu(0) & =\mu(a)=1, \\
\mu(b) & =0.7, \\
\mu(c) & =0.6, \\
\mu(d) & =0.8, \\
\mu\left(a^{\prime}\right) & =\mu\left(b^{\prime}\right)=\mu\left(c^{\prime}\right)=\mu\left(d^{\prime}\right)=\mu(1)=0.2 .
\end{aligned}
$$

Then, it is easy to see that $\mu$ is an $L$-fuzzy ideal of $Q$ and $a$ ' is a $\mu$-atom with respect to $\alpha=0.6$ in $[0,1]$.

Lemma 9. There always exists a $\mu$-atom for every proper $L$-fuzzy ideal $\mu$ in a poset $Q$ satisfying DCC with respect to some $\alpha$ in $L$.

Proof. Let $Q$ be a poset satisfying DCC and $\mu$ be a proper $L$-fuzzy ideal of $Q$. Then, there exists $a \in Q$ such that $\mu(a) \neq 1$. This implies that there exists $\alpha \in L$ such that $\alpha \Varangle \mu(a)$. Put $I=\{x \in Q: \mu(x) \geq \alpha\}$. Then, since $a \notin I, I$ is a proper ideal of $Q$ and $Q-I$ is a nonempty subset of $Q$. Since $Q$ is satisfying DCC, $Q-I$ has a minimal element, say $i$, such that $i \leq a$. Now we claim that $i$ is a $\mu$-atom with respect to $\alpha$. Since $i \in Q-I$, we have $\alpha \Varangle \mu(i)$. Let $x<i$. Then, by the minimality of $i, x \notin Q-I$ and hence $\mu(x) \geq \alpha$. Hence, the claim is true.

Remark 3. Lemma 9 gives a guarantee that if $\mu$ is an $L$-fuzzy ideal of a poset $Q$ satisfying DCC and $\alpha \not \mu(a)$ for some $a \in Q$ and $\alpha \in L$, then there exists a $\mu$-atom $i$ in $Q$ with respect to $\alpha$ such that $i \leq a$.

Lemma 10. Any two distinct $\mu$-atoms of an L-fuzzy ideal $\mu$ of a poset $Q$ with respect to $\alpha \in L$ are incomparable.

Proof. Let $\mu$ be an $L$-fuzzy ideal of $Q$ and $i$ and $j$ be any two distinct $\mu$-atoms with respect to $\alpha \in L$. Then, by definition, we have $\alpha \not \mu(i)$ and $\mu(x) \geq \alpha$ whenever $x<i$ and $\alpha \not \mu \mu(j)$ and $\mu(y) \geq \alpha$ whenever $y<j$. Now we show that $i$ and $j$ are incomparable. Suppose not. Then $i<j$ or $j<i$, i.e., $\mu(i) \geq \alpha$ or $\mu(j) \geq \alpha$, which is a contradiction to the fact that $\alpha \Varangle \mu(i)$ and $\alpha \not \mu(j)$. Hence, $i$ and $j$ are incomparable.

Remark 4. From Lemma 10, we can deduce that if $i$ and $j$ are $\mu$-atoms in a poset $Q$ with respect to some $\alpha$ in $L$ such that $i \leq j$, then $i=j$.

Lemma 11. Let $\mu$ be an $L$-fuzzy semiprime ideal of a poset $Q$ satisfying DCC. Then, $\mu: i$ is a $u$-L-fuzzy ideal for every $\mu$-atom $i$ in $Q$ with respect to 1 in $L$.

Proof. Let $i$ be a $\mu$-atom in $Q$ with respect to 1 in $L$. Since $\mu$ is an $L$-fuzzy semiprime ideal, by Theorem $2, \mu: i$ is an $L$-fuzzy ideal of $Q$. Now we show that $\mu: i$ is a $u$ - $L$-fuzzy ideal. Suppose on the contrary that $\mu: i$ is not a $u$ - $L$-fuzzy ideal. Then, there exist $a, b \in Q$ such that

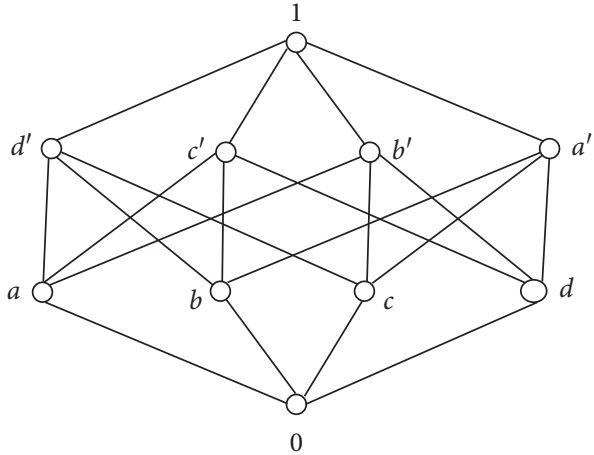

FIgURe 3: A distributive poset in which every L-fuzzy ideal need not be an L-fuzzy semiprime ideal.

$$
(\mu: i)(a) \wedge(\mu: i)(b) \not(\mu: i)(x) \forall x \in(a, b)^{u} .
$$

This implies that there exists $y \in(i, x)^{l}$ such that

$$
(\mu: i)(a) \wedge(\mu: i)(b) \not \mu(y) \forall x \in(a, b)^{u} .
$$

Thus, by Remark 3 , there exists a $\mu$-atom, say $j$, with respect to $\alpha=(\mu: i)(a) \wedge(\mu: i)(b)$ such that $j \leq y$. Since $j \leq y$ and $y \in(i, x)^{l}$, we have $j \leq i$ and hence $\mu(j) \geq \mu(i)$. This implies that $\alpha \nless \mu(i)$. Again, let $z<i$. Then, $\mu(z)=1 \geq \alpha$. Therefore, $i$ is also a $\mu$-atom with respect to $\alpha$. Also since $j \leq y \leq i$, by Remark 4 , we have $j=y=i$. This implies that $i \in(i, x)^{l}$ and hence $i \leq x$ for all $x \in(a, b)^{u}$ and so $i \in(a, b)^{u l}$. Since $\mu: i$ is an ideal, we have

$$
\alpha=(\mu: i)(a) \wedge(\mu: i)(b) \leq(\mu: i)(i)=\mu(i) .
$$

which is a contradiction to the fact that $\alpha \not \mu(i)$. Therefore, $\mu: i$ is a $u$ - $L$-fuzzy ideal.

Theorem 4. Let $\mu$ be an L-fuzzy ideal of a poset $Q$ satisfying DCC. Then, $\mu$ is an L-fuzzy semiprime ideal of $Q$ if and only if $\mu: i$ is an $L$-fuzzy ideal, in fact, an L-fuzzy prime ideal of $Q$ for every $\mu$-atom $i \in Q$ with respect to 1 in $L$.

Proof. Let $\mu$ be an $L$-fuzzy semiprime ideal of a poset $Q$ satisfying DCC and $i$ is a $\mu$-atom in $Q$ with respect to 1 in $L$. Then, by Lemma $11, \mu: i$ is a $u$-L-fuzzy ideal. Now, we have to show that $\mu: i$ is an $L$-fuzzy prime ideal. Since $\mu(i) \neq 1$, by Lemma 8 , $\mu: i \neq \overline{1}$. Hence, $\mu: i$ is proper. Let $a, b \in Q$ and suppose that

$$
\inf \left\{(\mu: i)(x): x \in(a, b)^{l}\right\} \neq(\mu: i)(a) \text {. }
$$

Put $\alpha=\inf \left\{(\mu: i)(x): x \in(a, b)^{l}\right\}$. Since $(\mu: i)(a)=\inf$ $\left\{\mu(y): y \in(a, i)^{l}\right\}$, there exists $y_{1}$ in $(i, a)^{l}$ such that $\alpha \nless \mu$ $\left(y_{1}\right)$. Then, by Remark 3, there exists a $\mu$-atom, say $j$, in $Q$ with respect to $\alpha$ such that $j \leq y_{1}$. It is also clear that $i$ is also a $\mu$-atom with respect to $\alpha$. Since $j \leq y_{1} \leq i$, by Remark 4, we must have $j=y_{1}=i$, and therefore $i \leq a$, i.e., $(i, a)^{l}=i^{l}$. Thus, we have

$$
\begin{aligned}
\inf \left\{(\mu: i)(x): x \in(a, b)^{l}\right\} & =\inf \left\{\mu(y): y \in(i, a, b)^{l}\right\} \\
& =\inf \left\{\mu(y): y \in(i, b)^{l}\right\} \\
& =(\mu: i)(b) .
\end{aligned}
$$


This proves that $\mu: i$ is an $L$-fuzzy prime ideal for every $\mu$-atom $i \in Q$.

Conversely, suppose that $\mu: i$ is an $L$-fuzzy ideal for any $\mu$-atom $i$ with respect to 1 in $L$. Let $a, b, c \in Q$. Now, we claim that

$$
\begin{array}{r}
\inf \left\{\mu(x) \wedge \mu(y): x \in(a, b)^{l}, y \in(a, c)^{l}\right\} \leq \mu(z), \\
\text { for all } z \in\left\{a,(b, c)^{u}\right\}^{l} .
\end{array}
$$

Suppose not. Then, there exists $z_{1} \in\left\{a,(b, c)^{u}\right\}^{l}=$ $a^{l} \cap(b, c)^{u l}$ such that

$$
\inf \left\{\mu(x) \wedge \mu(y): x \in(a, b)^{l}, y \in(a, c)^{l}\right\} \not \mu\left(z_{1}\right) .
$$

Hence, by Remark 3, there exists a $\mu$-atom $j$ in $Q$ with respect to $\alpha=\inf \left\{\mu(x) \wedge \mu(y): x \in(a, b)^{l}, y \in(a, c)^{l}\right\}$ in $L$ such that $j \leq z_{1}$. Then, by hypothesis, $\mu: j$ is an $L$-fuzzy ideal. Again, since $(j, b)^{l} \subseteq(a, b)^{l}$ and $(j, c)^{l} \subseteq(a, c)^{l}$, we have

$$
\begin{aligned}
\alpha= & \inf \left\{\mu(x) \wedge \mu(y): x \in(a, b)^{l}, y \in(a, c)^{l}\right\} \\
= & \inf \left\{\mu(x): x \in(a, b)^{l}\right\} \wedge \inf \left\{\mu(y): y \in(a, c)^{l}\right\} \\
& \leq \inf \left\{\mu(x): x \in(j, b)^{l}\right\} \wedge \inf \left\{\mu(y): y \in(j, c)^{l}\right\} \\
= & (\mu: j)(b) \wedge(\mu: j)(c) \\
& \leq(\mu: j)(j)\left(\text { since } j \in(b, c)^{u l}\right) \\
= & \mu(j),
\end{aligned}
$$

which is a contradiction to the fact that $j$ is a $\mu$-atom with respect to $\alpha$. Therefore, $\mu$ is an $L$-fuzzy semiprime ideal of $Q$.

The following result gives another characterization for $L$-fuzzy semiprime ideals to be $L$-fuzzy prime.

Theorem 5. Every maximal L-fuzzy semiprime ideal of a poset $Q$ is an L-fuzzy prime ideal.

Proof. Let $\mu$ be a maximal $L$-fuzzy semiprime ideal of a poset $Q$, that is, maximal among all proper $L$-fuzzy semiprime ideals of a poset $Q$. Let $a, b \in Q$. Then, by Theorem 2, $\mu: b$ is an $L$-fuzzy semiprime ideal. Since $\mu \subseteq \mu: b$, by maximality of $\mu$, we have either $\mu=\mu: b$ or $\mu: b=\overline{1}$. If $\mu: b=\overline{1}$, then, by Lemma $8, \mu(b)=1$. Thus,

$$
\inf \left\{\mu(x): x \in(a, b)^{l}\right\}=(\mu: b)(a)=\overline{1}(a)=1=\mu(b) .
$$

Again if $\mu=\mu: b$, then we have $\inf \{\mu(x)$ : $\left.x \in(a, b)^{l}\right\}=(\mu: b)(a)=\mu(a)$. Thus, in either cases, we have

$$
\inf \left\{\mu(x): x \in(a, b)^{l}\right\}=\mu(a) \text { or } \mu(b), \quad \text { for all } a, b \in Q \text {. }
$$

Hence, $\mu$ is an $L$-fuzzy prime ideal of $Q$.

As a consequence, we have the following corollary.

Corollary 2. Let $\mu$ be a maximal L-fuzzy ideal of $Q$. Then, $\mu$ is an L-fuzzy semiprime ideal $Q$ if and only if $\mu$ is an $L$-fuzzy prime ideal.
The following is a characterization of an $L$-fuzzy ideal to be $L$-fuzzy prime ideal in terms of a $\mu$-atom in a poset $Q$ satisfying DCC.

Theorem 6. Let $\mu$ be an L-fuzzy ideal of a poset $Q$ satisfying $D C C$. Then, $\mu$ is an L-fuzzy prime ideal $Q$ if and only if $Q$ has exactly one $\mu$-atom with respect to some $\alpha$ in $L$.

Proof. Let $\mu$ be an $L$-fuzzy prime ideal of a poset $Q$ satisfying DCC. Since $\mu$ is proper, by Lemma 9 , there exists a $\mu$-atom in $Q$ with respect to some $\alpha$ in $L$. Now, we claim that $Q$ has exactly one $\mu$-atom with respect to $\alpha$ in $L$. Suppose not. Let $i, j \in Q$ be any distinct $\mu$-atoms in $Q$ with respect to $\alpha$ in $L$. Then, by Lemma $10, i, j$ are incomparable and $\mu(x) \geq \alpha$ for all $x<i$ and $\mu(y) \geq \alpha$ for all $y<j$. This implies that $\inf \left\{\mu(x): x \in(i, j)^{l}\right\} \geq \alpha$. Since inf $\left\{\mu(x): x \in(i, j)^{l}\right\}=\mu(i)$ or $\mu(j)$, we have $\mu(i) \geq \alpha$ or $\mu(j) \geq \alpha$, which is a contradiction. Therefore, $Q$ has exactly one $\mu$-atom with respect to $\alpha$ in $L$.

Conversely suppose that $Q$ has exactly one $\mu$-atom, say $i$, with respect to some $\alpha$ in $L$. Now, we show that $\mu$ is an $L$-fuzzy prime ideal. Since $\alpha \not \xi \mu(a)$, we have $\mu(i) \neq 1$ and hence $\mu$ is proper. Now, we show that for any $a, b \in Q$,

$$
\begin{aligned}
\inf \left\{\mu(x): x \in(a, b)^{l}\right\} & =\mu(a), \\
& \text { or } \mu(b) .
\end{aligned}
$$

Suppose not. Thus, there exist $a, b \in Q$ such that

$$
\begin{aligned}
& \inf \left\{\mu(x): x \in(a, b)^{l}\right\} \not \mu(a), \\
& \inf \left\{\mu(x): x \in(a, b)^{l}\right\} \not \mu(b) .
\end{aligned}
$$

Then, there exist $\mu$-atoms $i, j \in Q$ with respect to $\alpha=$ $\inf \left\{\mu(x): x \in(a, b)^{l}\right\}$ such that $i \leq a$ and $j \leq b$. Then, by hypothesis, we have $i=j$ and hence $i \in(a, b)^{l}$. Therefore $\alpha=\inf \left\{\mu(x): x \in(a, b)^{l}\right\} \leq \mu(a)$, which is a contradiction to the fact that $i$ is a $\mu$-atom with respect to $\alpha=\inf \left\{\mu(x): x \in(a, b)^{l}\right\}$. Therefore, $\mu$ is an $L$-fuzzy prime ideal.

Lemma 12. Let $\mu$ be a proper L-fuzzy ideal of a poset $Q$ satisfying DCC and $A=\{i \in Q: i$ is $a \mu-$ atom $\}$. Then, $\mu=\cap_{i \in A}: \mu: i$.

Proof. We show that $\cap_{i \in A} \mu: i \subseteq \mu$ as the converse inclusion always holds. Suppose that $\cap_{i \in A} \mu: i \nsubseteq \mu$. This implies that there exists $a \in Q$ such that $\left(\cap_{i \in A} \mu: i\right)(a) \not \mu(a)$. Thus, there exists a $\mu$-atom $j \in Q$ with respect to $\alpha=\left(\cap_{i \in A} \mu: i\right)(a)$ such that $j \leq a$.

Then, we have $j \in A$, and hence,

$$
\begin{aligned}
\left(\bigcap_{i \in A}: \mu\right)(a) & \leq(\mu: j)(a) \\
& =\inf \left\{\mu(x): x \in(j, a)^{l}\right\} \\
& =\inf \left\{\mu(x): x \in j^{l}\right\} \\
& =\mu(j),
\end{aligned}
$$


which is a contradiction to the fact that $j$ is a $\mu$-atom with respect to $\alpha=\left(\cap_{i \in A} \mu: i\right)(a)$. Hence, $\alpha=\left(\cap_{i \in A} \mu: i\right) \mu$. Therefore, $\left(\cap_{i \in A} \mu: i\right) \subseteq \mu$.

Lemma 13. The intersection of any nonempty family of $L$-fuzzy prime ideals of $Q$ is an L-fuzzy semiprime ideal $Q$.

Proof. Let $\left\{\mu_{i}: i \in \Delta\right\}$ be a nonempty family of $L$-fuzzy prime ideals of $Q$. Put $\mu=\cap_{i \in \Delta} \mu_{i}$. Then, clearly, $\mu$ is an $L$-fuzzy ideal of $Q$. Let $a, b, c \in Q$ and $z \in\left\{a,(b, c)^{u}\right\}^{l}$. Now,

$$
\begin{aligned}
& \inf \left\{\mu(x) \wedge \mu(y): x \in(a, b)^{l}, y \in(a, c)^{l}\right\} \\
& =\inf \left\{\mu(x): x \in(a, b)^{l}\right\} \wedge \inf \left\{\mu(y): y \in(a, c)^{l}\right\} \\
& \leq \inf \left\{\mu_{i}(x): x \in(a, b)^{l}\right\} \wedge \inf \left\{\mu_{i}(y): y \in(a, c)^{l}\right\}, \\
& \text { for each } i \in \Delta .
\end{aligned}
$$

$=\mu_{i}(a)$ or $\mu_{i}(b) \wedge \mu_{i}(c), \quad$ for each $i \in \Delta$.

$\leq \mu_{i}(z), \quad$ for each $i \in \Delta$.

This implies that

$$
\begin{aligned}
& \inf \left\{\mu(x) \wedge \mu(y): x \in(a, b)^{l}, y \in(a, c)^{l}\right\} \\
& \quad \leq\left(\bigcap_{i \in \Delta}: \mu_{i}\right)(z)=\mu(z), \quad \text { for all } z \in\left\{a,(b, c)^{u}\right\}^{l} .
\end{aligned}
$$

Therefore, $\mu=\cap_{i \in \Delta} \mu_{i}$ is an $L$-fuzzy semiprime ideal $Q$.

As an immediate consequence of Theorem 4, Lemma 12, and Lemma 13 in the case of posets satisfying DCC, we obtain the following result.

Theorem 7. Let $\mu$ be a proper L-fuzzy ideal of a poset $Q$ satisfying DCC. Then, $\mu$ is an L-fuzzy semiprime ideal of $Q$ if and only if $\mu$ is expressed as an intersection of L-fuzzy prime ideals of $Q$.

In the following, we characterize the distributive posets in terms of $L$-fuzzy semiprime ideals in the following results.

Theorem 8. A poset $Q$ is distributive if and only if $\chi_{(x]}$ of $Q$ is an L-fuzzy semiprime ideal of $Q$, for each $x \in Q$.

Proof. Suppose that $Q$ is distributive poset and $x \in Q$. Now to show $\chi_{(x]}$ is an $L$-fuzzy semiprime ideal of $Q$, by Corollary 1 , it is enough to show that $(x]$ is a semiprime ideal of $Q$. Let $a, b, c \in Q$ such that $(a, b)^{l} \subseteq(x]$ and $(a, c)^{l} \subseteq(x]$. Let $z \in\left\{a,(b, c)^{u}\right\}^{l}$. Then, $z \leq a$ and $z \in(b, c)^{u l}$. This implies that

$$
z^{l}=\left\{z,(b, c)^{u}\right\}^{l}=\left\{(z, b)^{l},(z, c)^{l}\right\}^{u l} .
$$

Since $z \leq a$, we have $(z, b)^{l} \subseteq(a, b)^{l} \subseteq(x]$ and $(z, c)^{l} \subseteq$ $(a, c)^{l} \subseteq(x]$.

This implies that $(z, a)^{l} \cup(z, b)^{l} \subseteq(x]$. Thus, $z \in z^{l}=$ $\left\{(z, b)^{l},(z, c)^{l}\right\}^{u l} \subseteq(x]^{u l}=(x]$ and hence, $\left\{a,(b, c)^{u}\right\}^{l} \subseteq(x]$.

Therefore, $\chi_{(x]}$ is an $L$-fuzzy semiprime ideal of $Q$.

Conversely, suppose that $\chi_{(x]}$ is an $L$-fuzzy semiprime ideal of $Q$ for each $x \in Q$. Then, by Corollary 1 , it is clear that $(x]$ is semiprime ideal of $Q$ for each $x \in Q$. Let $a, b, c \in Q$. It is enough to prove that $\left\{a,(b, c)^{u}\right\}^{l} \subseteq\left\{(a, b)^{l},(a, c)^{l}\right\}^{u l}$, as the converse inclusion is always true. Now let $x \in\left\{a,(b, c)^{u}\right\}^{l}$ and $y \in\left\{(a, b)^{l},(a, c)^{l}\right\}^{u}$. We claim that $x \leq y$. Indeed, since $\left\{(a, b)^{l},(a, c)^{l}\right\}^{u l} \subseteq y^{l}$, we have

$$
\begin{aligned}
& (a, b)^{l} \subseteq y^{l}=(y], \\
& (a, c)^{l} \subseteq y^{l}=(y] .
\end{aligned}
$$

Then, by semiprimeness of $(y]$, we conclude that $x \in\left\{a,(b, c)^{u}\right\}^{l} \subseteq(y]$. Hence, $x \leq y$ for all $y \in\left\{(a, b)^{l}\right.$, $\left.(a, c)^{l}\right\}^{u}$. Therefore, $x \in\left\{(a, b)^{l},(a, c)^{l}\right\}^{u l}$, and hence,

$$
\left\{a,(b, c)^{u}\right\}^{l} \subseteq\left\{(a, b)^{l},(b, c)^{l}\right\}^{u l} .
$$

This proves that $Q$ is a distributive poset.

Note that in a distributive poset every $L$-fuzzy ideal need not be an $L$-fuzzy semiprime ideal. Consider the distributive poset $Q$ depicted in Figure 3.

Define a fuzzy subset $\mu: Q \longrightarrow[0,1]$ by

$$
\begin{aligned}
\mu(0) & =\mu(a)=1, \\
\mu\left(a^{\prime}\right) & =\mu\left(b^{\prime}\right)=\mu\left(c^{\prime}\right)=\mu\left(d^{\prime}\right)=\mu(1)=0.2, \\
\mu(b) & =0.6, \\
\mu(c) & =0.5, \\
\mu(d) & =0.7 .
\end{aligned}
$$

Then, $\mu$ is an $L$-fuzzy ideal but not an $L$-fuzzy semiprime ideal as $d^{\prime}, c^{\prime}, b^{\prime} \in Q$ and $d^{\prime} \in\left\{d^{\prime},\left(b^{\prime}, c^{\prime}\right)^{u}\right\}^{l}$ but

$$
\begin{aligned}
& \inf \left\{\mu(x) \wedge \mu(y): x \in\left(d^{\prime}, c\right)^{l}, y \in\left(d^{\prime}, b^{\prime}\right)^{l}\right\} \\
& \quad=0.5 \nless 00.2=\mu\left(d^{\prime}\right) .
\end{aligned}
$$

As an immediate consequence of Theorem 7 and Theorem 8 , we have the following corollary.

Corollary 3. Let $Q$ be a poset satisfying DCC. Then, $Q$ is distributive if and only if, for every $x \in Q, \chi_{(x]}$ is representable as an intersection of $L$-fuzzy prime ideals of $Q$.

\section{Conclusion}

In this work, we introduce the notions of $L$-fuzzy semiprime ideal in general posets. Characterizations of $L$-fuzzy semiprime ideals in posets as well as characterizations of an $L$-fuzzy semiprime ideal to be $L$-fuzzy prime ideal are obtained by introducing the concept $\mu$-atom elements in a poset. Also, $L$-fuzzy prime ideals in a poset are characterized. Our future work will focus on the relations between the $L$-fuzzy semiprime (resp., $L$-fuzzy prime) ideals of a poset and the $L$-fuzzy semiprime (resp., $L$-fuzzy prime) of the lattice of all ideals of a poset. We will also extend and prove an analogue of Stone's theorem for finite posets using L-fuzzy semiprime ideals.

\section{Data Availability}

No data were used to support the results of this study. 


\section{Conflicts of Interest}

The authors declare that there are no conflicts of interest regarding the publication of this paper.

\section{Acknowledgments}

This research work was partially supported by the research grant from the College of Science, Bahir Dar University.

\section{References}

[1] L. A. Zadeh, "Fuzzy sets," Information and Control, vol. 8, no. 3, pp. 338-353, 1965.

[2] A. Rosenfeld, "Fuzzy groups," Journal of Mathematical Analysis and Applications, vol. 35, no. 3, pp. 512-517, 1971.

[3] V. N. Dixit, R. Kumar, and N. Ajmal, "On fuzzy rings," Fuzzy Sets and Systems, vol. 49, no. 2, pp. 205-213, 1992.

[4] V. N. Dixit, R. Kumar, and N. Ajmal, "Fuzzy ideals and fuzzy prime ideals of a ring," Fuzzy Sets and Systems, vol. 44, no. 1, pp. 127-138, 1991.

[5] U. M. Swamy and K. L. N. Swamy, "Fuzzy prime ideals of rings," Journal of Mathematical Analysis and Applications, vol. 134, no. 1, pp. 94-103, 1988.

[6] U. Acar, "On L-fuzzy prime submodules," Hacettepe Journal of Mathematics and Statistics, vol. 34, pp. 17-25, 2005.

[7] R. Ameri and R. Mahjoob, "Spectrum of prime -submodules," Fuzzy Sets and Systems, vol. 159, no. 9, pp. 1107-1115, 2008.

[8] B. B. Makamba and V. Murali, "On prime fuzzy submodules and radicals," The Journal of Fuzzy Mathematics, vol. 8, no. 4, pp. 831-843, 2000.

[9] P. Das, "Fuzzy vector spaces under triangular norms," Fuzzy Sets and Systems, vol. 25, no. 1, pp. 73-85, 1988.

[10] A. K. Katsaras and D. B. Liu, "Fuzzy vector spaces and fuzzy topological vector spaces," Journal of Mathematical Analysis and Applications, vol. 58, no. 1, pp. 135-146, 1977.

[11] N. Ajmal and K. V. Thomas, "Fuzzy lattices," Information Sciences, vol. 79, no. 3-4, pp. 271-291, 1994.

[12] B. A. Alaba and W. Z. Norahun, " $\alpha$ - fuzzy ideals and space of prime - $\alpha$ fuzzy ideals in distributive lattices," Annals of Fuzzy Mathematics and Informatics, vol. 17, no. 2, pp. 147-163, 2019.

[13] Y. Bo and W. Wangming, "Fuzzy ideals on a distributive lattice," Fuzzy Sets and Systems, vol. 35, no. 2, pp. 231-240, 1990.

[14] B. B. N. Koguep and C. Lele, "On fuzzy prime ideals of lattices," SJPAM, vol. 3, pp. 1-11, 2008.

[15] U. M. Swamy and D. V. Raju, "Fuzzy ideals and congruences of lattices," Fuzzy Sets and Systems, vol. 95, no. 2, pp. 249-253, 1998.

[16] B. A. Alaba and G. M. Addis, "L-fuzzy ideals in universal algebras," Annals of Fuzzy Mathematics and Informatics, vol. 17, no. 1, pp. 31-39, 2019.

[17] B. A. Alaba and G. M. Addis, " $L$-fuzzy prime ideals in universal algebras," Advances in Fuzzy Systems, vol. 2019, Article ID 5925036, 7 pages, 2019.

[18] B. A. Alaba and T. G. Alemayehu, "Closure fuzzy ideals of MSalgebras," Annals of Fuzzy Mathematics and Informatics, vol. 16, pp. 247-260, 2018.

[19] B. A. Alaba and T. G. Alemayehu, "Fuzzy ideals in demipseudocomplemented MS-algebras," Annals of Fuzzy Mathematics and Informatics, vol. 18, pp. 123-143, 2019.

[20] B. A. Alaba and W. Z. Norahun, "Fuzzy ideals and fuzzy filters of pseudo-complemented semilattices," Advances in Fuzzy Systems, vol. 2019, Article ID 4263923, 13 pages, 2019.
[21] B. A. Alaba and G. M. Addis, "L-fuzzy semi-prime ideals in universal algebras," The Korean Journal of Mathematics, vol. 27, no. 2, pp. 327-340, 2019.

[22] N. Kehayopulu, X. Y. Xie, and M. Tsingelis, "A characterization of prime and semiprime ideals of semigroups in terms of fuzzy subsets," Soochow Journal of Mathematics, vol. 27, no. 2, pp. 139-144, 2001.

[23] R. Kumar, "Fuzzy ideals and fuzzy semiprime ideals: some ring theoretic analogues," Information Sciences, vol. 66, no. 12, pp. 43-52, 1992.

[24] H. V. Kumbhojkar and M. S. Bapat, "On semiprime fuzzy ideals," Fuzzy Sets and Systems, vol. 60, no. 2, pp. 219-223, 1993.

[25] N. Kuroki, "Fuzzy semiprime ideals in semigroups," Fuzzy Sets and Systems, vol. 8, no. 1, pp. 71-79, 1982.

[26] M. M. Zahedi, "A note on L-fuzzy primary and semiprime ideals," Fuzzy Sets and Systems, vol. 51, no. 2, pp. 243-247, 1992.

[27] J. A. Goguen, "L-fuzzy sets," Journal of Mathematical Analysis and Applications, vol. 18, no. 1, pp. 145-174, 1967.

[28] G. Birkhoff, Lattice Theory, American Mathematical Society Colloquium Publications, Providence, RI, USA, 1961.

[29] O. Frink, "Ideals in partially ordered sets," The American Mathematical Monthly, vol. 61, no. 4, pp. 223-234, 1954.

[30] R. Halaś, "Annihilators and ideals in ordered sets," Czechoslovak Mathematical Journal, vol. 45, no. 120, pp. 127-134, 1995.

[31] P. V. Venkataranasimhan, "Pseudo-complements in posets," Proceedings of the American Mathematical Society, vol. 28, no. 1, pp. 9-17, 1971.

[32] P. V. Venkataranasimhan, "Semi-ideals in posets," Mathematische Annalen, vol. 185, no. 4, pp. 338-348, 1970.

[33] M. Ernè, Verallgemeinerungen der Verbandstheorie II: m-Ideale in halbgeordneten Mengen und Hüllenräumen, University of Hannover, Hannover, Germany, 1979.

[34] R. Halaś and J. Rachůnek, "Polars and prime ideals in ordered sets," Discussiones Mathematicae: Algebra and Stochastic Methods, vol. 15, pp. 43-59, 1995.

[35] V. S. Kharat and K. A. Mokbel, "Primeness and semiprimeness in posets," Mathematica Bohemica, vol. 134, no. 1, pp. 19-30, 2009.

[36] B. A. Alaba, M. A. Taye, and D. A. Engidaw, "L-Fuzzy ideals of a poset," Annals of Fuzzy Mathematics and Informatics, vol. 16, pp. 285-299, 2018.

[37] B. A. Alaba, M. Alamneh, and D. Abeje, "L-Fuzzy filters of a poset," International Journal of Computing Science and Applied Mathematics, vol. 5, no. 1, pp. 23-29, 2019.

[38] B. A. Alaba, M. A. Taye, and D. A. Engidaw, "L-fuzzy prime ideals and maximal L-fuzzy ideals of a poset," Annals of Fuzzy Mathematics and Informatics, vol. 18, no. 1, pp. 1-13, 2019.

[39] U. M. Swamy and D. Viswanadha Raju, "Algebraic fuzzy systems," Fuzzy Sets and Systems, vol. 41, no. 2, pp. 187-194, 1991.

[40] U. M. Swamy and D. Viswanadha Raju, "Irreducibility in algebraic fuzzy systems," Fuzzy Sets and Systems, vol. 41, no. 2, pp. 233-241, 1991.

[41] B. A. Davey and H. A. Priestley, Introduction to Lattices and Order, Cambridge University Press, Cambridge, UK, 1990.

[42] G. Grätzer, General Lattice Theory, Academic Press, Cambridge, MA, USA, 1978.

[43] J. Larmerová and J. Rachůnek, "Translations of distributive and modular ordered sets," Acta Universitatis Palackianae Olomucensis, Facultas Rerum Naturalium / Mathematica, vol. 91, pp. 13-23, 1988.

[44] Y. Rav, "Semiprime ideals in general lattices," Journal of Pure and Applied Algebra, vol. 56, no. 2, pp. 105-118, 1989. 